\title{
Design a network for Iraqi Universities E-Learning Centers
}

\author{
Ali Hussein Mousa ${ }^{1}$, Amer Saleem Flayyeh Elameer ${ }^{2}$ \\ University of Information Technology and Communications (UOITC), Baghdad, Iraq ${ }^{1}$ \\ Informatics Institute for Postgraduate Studies ${ }^{2}$
}

\begin{abstract}
The e-Learning occupies a high interest in the international universities and academic institutes and given priority by departments in those educational institutions, what we are watching in Iraq with the development of information, communications, wireless networking technology and multimedia push in the direction of the adoption of e-Learning, where it could provide students with learning and knowledge by employing these technologies and continuous manners, regarding less time and place through the delivery, organizing educational materials electronically, so Iraqi student can review and learn from them, either side by side and directly with a lecturer, or through distance learning.[1] As long as the e-Learning is a form of distance learning, so it can be considered as a way to teach using the modern communication mechanisms, such as computers (of all kinds: personal, clients, servers) and networks and multimedia and Internet web applications to deliver information to students as soon as possible and less expensive and to enable the educational process to be management, arrangement, measuring and evaluating their performance. So from the above shows the importance of creating an electronic network for Iraqi universities and academic institutes that accommodates all abilities available to them to provide appropriate environments of software with tools, devices and mechanisms of linking to the environment according to the features of each device and its mission and its location within the network, taking into consideration the possibility of the development of specifications of the devices and probability of Network expansion in the future.[2]
\end{abstract}

Keywords: LMS (Learning Management System), LCMS (Learning Content Management System), SCORM (Sharable Content Object Reference Model), SAN(storage area network), CCTV (closed circuit television), RHEL (Red Hat Enterprise Linux), SNMP (Simple Network Management Protocol), ECBF(Explicit, Compressed, Beam forming), WebCT (Web Course Tools).

\section{INTRODUCTION}

This era is characterized by the rapid changes resulting technologies of the computer and the global network of from scientific and technological advances and information and variant Multi Media such as CD-ROMs, information technology, so it became necessary to keep up educational software, and e-mail, and Yards dialogue and with the educational process for these changes to address discussion and virtual classrooms.[2] the problems that may arise from them, such as the large number of information and increase the number of students and the lack of teachers and distances.

These changes have led to the emergence of patterns of Network design should ensure that e-Learning center many ways to teach, particularly in the area of individual components and devices can modify and scalable to the instruction or self in which the learner is going according demands of new services, and to support e - Learning to his energy and ability and speed of learning, according center with more efficiency, the design must consider to what he has of experience and previous skills, so as a in mind that the networks should be available nearly solutions against of these changes, there appeared the 100 percent of the time and guarantee the reliability concept of programmed instruction, and the concept of of information while the network security will be able to Assistant Education Computer and the concept of distance automatically correcting unexpected security bugs.[1] learning, in which students learn anywhere without the need for a teacher on a permanent basis.[1]

Depending on both "hierarchical network design With the advent of the technological revolution in principles" and "organized design methodology ", to information technology, which has made the world a build a manageable, flexible, extendable and supportable small village has increased the need to share experiences e-Learning center.

with others, and the need of the student multivendor Computers and information networks are critical to the environments that it is useful in research and self- success of businesses, both large and small, they connect development, there appeared the concept of e-learning, people, support applications and services , and provide which is a method of teaching methods in the delivery of access to the resources that keep the businesses running, information to the learner, it depends on modern to meet the daily requirements of businesses, networks 
Vol. 4, Issue 2, February 2017

themselves are becoming quite complex and more developed.

\section{The structure of an e-learning center's Network:}

The structure of the Network should be chosen before considering the visuals, the information architecture used to describe the planning of a Network structure could be as follow[3]:

\section{(A)-Link Balancer}

Is a network machine that distributes in-bound and outbound traffic to and from multiple Internet Service Provider (ISP), link load balancers are typically located between gateway routers and firewall.

\section{(B)-Firewall}

Firewall is a network security system designed to prevent unauthorized access to or from a private network, firewalls can be implemented in hardware \& software, or a combination of both.

\section{(C)-Core Switch}

A core switch is a high -capacity switch generally positioned within the backbone or physical core of a network. Core switches serve as the gateway to a wide area network (WAN) or the Internet - they provide the final aggregation point for the network and allow multiple aggregation modules to work together, a core switch is also known as a tandem switch or a backbone switch, in a public WAN, a core switch interconnects edge switches that are positioned on the edges of related networks.

\section{(D)-Gigabit Switch (Ethernet)}

A "central hub" wired to every computer and network device in an Ethernet. In the home and small office, an Ethernet switch is built into the router .Gigabit Ethernet (10/100/1000) switches support 1 Gbps and also 10 Mbps and $100 \mathrm{Mbps}$ for compatibility with lower-speed devices. Older 10/100 switches cannot handle Gigabit speeds; however, all Ethernet devices sense the speed of the connection and transmit at the highest common denominator.

\section{(E)-Fiber Channel Switch}

In the computer storage field, a Fiber Channel switch is a network switch compatible with the Fiber Channel (FC) protocol. It allows the creation of a Fiber Channel fabric, which is currently the core component of most storage area networks (SAN)

\section{(F)-Storage Area Network}

SAN (storage area network) is a high- speed network of storage devices that also connects those storage devices with servers, it provides block-level storage that can be accessed by the applications running on any networked servers.

\section{(G)-Wireless Access Point (AP)}

A hardware device or a computer's software that acts as a communication hub for users of a wireless device to connect to a wired LAN, access points devices are important for providing heightened wireless security and for extending the physical range of service.

\section{(H)-IP Camera}

Is a type of digital video camera commonly employed for surveillance, and which, unlike analog closed circuit television (CCTV) cameras, can send and receive data via a computer network and the Internet.

\section{(I)-Web + File + Email Server}

Is put to use for filing and print sharing services and the software used are Red Hat Linux Enterprise ( RHEL ) Version 5, Apache Web Server, Nagios Network Monitoring Tools, controlling the e-education activities by its software Moodle, LAMS and JUSUR.

This server in the network is the e-Learning center web server, student web server, and the Moodle LAMS and JUSUR server, this server will be running Windows Server 2003 Enterprise, Internet Information Services Web Server, Cacti Network Monitor Tool, and Moodle, the function of this server is to provide a location for faculty and students to create personal web pages, this server will also allow for the hosting of Moodle, a highly configurable resource for distance learning, cacti will serve as the network management and monitoring tool, it will use its own generated scripts as well as SNMP to monitor status of network devices that also support the Simple Network Management Protocol (SNMP) protocol ( (SNMP) is an "Internet-standard protocol for managing devices on IP networks ), this configuration requires PHP (Hypertext Preprocessor and it is free software released under the PHP License) and MySQL to be installed on the server system in order for these tools to function, this server does have an alternate configuration, server 2003 was chosen for the ease of manageability, and the ease of security management (monitoring for new security vulnerabilities), as well as an easier patch deployment and management. If there is no desire for a homogeneous network, Red Hat Enterprise Linux could be used, if this OS is used, Apache web server can be used to run Moodle and Cacti, Open NMS or Nagios can still be used to manage the network[1]

(J)-Database, Web, Application, File, Video Management, Standby Server

Is the web server and also act as the SSH server (Secure Shell or SSH is a network protocol that allows data to be exchanged using a secure channel between two or more computer over a network) for the network, and the software used are Microsoft Windows Server 2012 R2 Foundation, Microsoft Hyper-V 2008, Fedora Core 12, Microsoft Windows 10 Pro Education, and Microsoft Office Suite 2013 Professional and it will be also as a part of Active Directory and will be grouped with server 4 to supply an operating system interface to learners remotely accessing the virtual network. This server in the network is the first of the Virtual PC servers and will run Windows Server 2008 R2 Enterprise, and Virtual Server 
2005 R2. The virtual server will be capable to execute Fedora Core 6 as Virtual OS, Windows XP Professional as Virtual OS, Windows Server 2008 R2 Enterprise.

Initiative as Virtual OS, and Microsoft Office Suite 2007 Professional on Virtual O S, the responsibilities of this server are: to be an associate of Active Directory, grouped with the 4th server, and provide OS interface to learners remotely accessing the virtual network.[4]

\section{(K)-Domain Controller + Virtualization Server}

Is the domain controller, DNS server, and a backup server, it will be the domain controller (DC) and SharePoint Portal 2010 Server, the software is Microsoft Windows Server 2012 R2 Foundation Edition. It will work as the primary domain controller in active directory; it will also supply Dynamic Host Configuration Protocol (DHCP) to the network and turn as a gateway to the virtual network. This server will run Microsoft Windows Server 2008 R2, will be the Share Point Portal 2010 Server, and provides web services to the university colleges and departments. This server will be the domain controller and SharePoint Portal Server and it will run Windows Server 2003 R2 NT 5.2 version 2006 and SharePoint Portal Server. It will furnish web services through SharePoint, and will be the gateway to the virtual network. This server will act as the primary domain controller in active directory and will provide DHCP services to the network as well as administer user accounts through active directory.[3]

\section{(L)-PC / Workstation}

Workstations generally come with a large, highresolution graphics screen, at least $64 \mathrm{MB}$ (megabytes) of RAM, built-in networksupport, and a graphical user interface. Most workstations also have a mass storage device such as a disk drive, but a special type of workstation, called a diskless workstation, comes without a disk drive. The most common operating systems for workstations are UNIX and Windows NT.

\section{(M)-Video Server}

The term "video server" refers to a network-attached server for video that is connected to a computer network like a LAN. A video server can deliver live video, automatically or on request, to a browser or other professional security applications. Security systems have traditionally been based on analog CCTV (closed-circuit television) technology. Video servers digitize analog video sources and distribute digital video over an IP network, Turninganalog cameras into IP cameras, a video server can also be connected via a modem for access over a phone or ISDN line.

\section{(N)-Video Conference}

Conduct a conference between two or more participants at different sites by using computer networks to transmit audio and video data. For example, a point-to-point (twoperson) video conferencing system works much like a video telephone. Each participant has a video camera, microphone, and speakers mounted on his or her computer. As the two participants speak to one another, their voices are carried over the network and delivered to the other's speakers, and whatever images appear in front of the video camera appear in a window on the other participant's monitor.

\section{RESULTS AND DISCUSSION}

Based on the above, we can collect the components to generate the network hardware layout of the e-learning center to show the right position of each device according to the purpose of use and duty at the e-Learning center, and to Exploit all the technical features of each item for more efficiency and performance while operating the network, as shown in the Figure bellow:

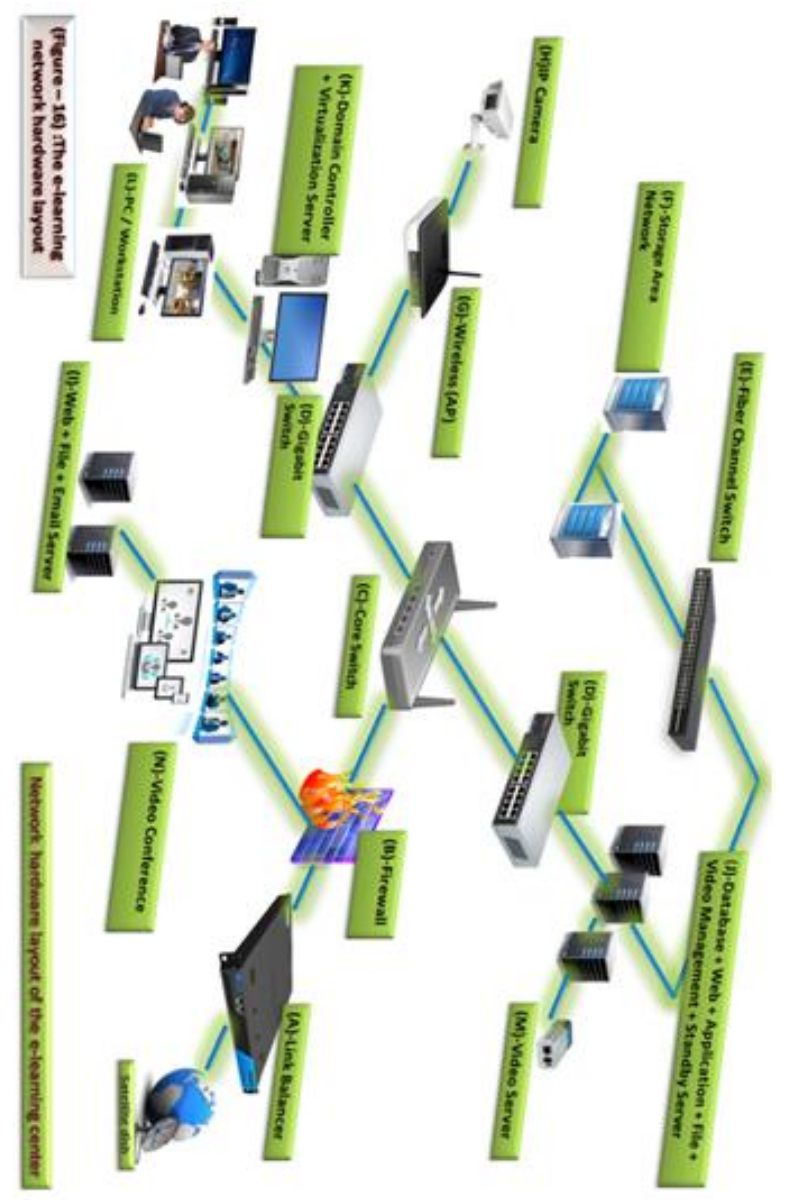

The result of research

Here the result in which e-Learning has transformed the landscape of learning and development. When compared to the traditional mode of classroom learning, there is clear evidence that e-Learning brings:

\section{It's what learners want - really}

The recent report from towards maturity on the gap between corporate learning and what learners actually want highlights that, when given a choice, learners want mobile, relevant, personalized and self-paced content at a point of need. What they get is often a little different with too much emphasis on face-to-face and long courses. 


\section{Faster delivery}

At a time when change is faster than ever, a key advantage of e-Learning is that it has quicker delivery cycle times than traditional classroom.

\section{Lower costs}

Because of the speed and ease in which e-Learning is delivered, the costs of learning and development for an organization are drastically reduced.

\section{More effective learning}

Our case studies show time and again those e-Learning courses can have a positive and direct impact on an organization's profitability by making it easy to learn and digest information.

\section{Lower environmental impact}

By providing an alternative to the paper-based learning and testing of traditional classrooms, e-Learning is an effective way for organizations to significantly reduce their carbon footprint, and it consider as a green network

\section{CONCLUSION}

To obtain a typical vision on this research we have to focus on the reasons that lead us to research and the benefits of using e-Learning Tools

\section{4/7 Accessibility:}

The e-Learning materials may be made accessible to the workforce throughout the day and around the week. Lastly, given that present day employees work out of different time zones, a learning tool that is available 24/7 makes it possible for learners without a constraint on resources.

\section{Improved Pedagogy:}

Studies have shown that gamification enhances learner engagement and improves retention, it is not just gamification, and e-Learning also helps with the use of personalized study materials and interactive formats.

\section{Enhanced Collaboration and Reach.}

With e - Learning, it is possible to gain instant reach to staff and trainers from all parts of the world, besides enabling teams from various geographies to collaborate on problem-solving challenges in real-time.

\section{Greener \& Cost-Effective}

Typically, money is spent on trainer and employee commutation, classroom rentals, infrastructure rentals, trainer cost per hour and paper and documentation costs, e - Learning is not only cheaper but also greener, in terms of costs, businesses no longer have to spend on commutation, and classroom \& infrastructure rentals.

\section{Suitable For Millennial}

With eLearning, the knowledge is always available at the Learner's disposal this gives them better access to subjects they are interested in and not necessarily those that would benefit them at life. Not only does this benefit the learner in what does the learner is specialist with, but is also increase the General knowledge of the learner mind.

\section{REFERENCES}

[1] Amer Saleem Elameer, Rozhan M.Idrus \& Atyaf Alhadithi (2011). Orbital E-Education Framework for the University of Mustansiriyah (UoMust), Baghdad, Iraq.In A. Zaharim et al. (Eds.). Recent Researches in Education, WSEAS Press, pp. 4348 [ISBN: 978-1-61804-040-4] [ISI Listed]

[2] Hansen, D. J. (2003). Book review: E-Learning: Strategies for Delivering Knowledge in the Digital Age (Author: M. Rosenberg). Educational Technology \& Society, 6(3), 80-81 (ISSN 1436-4522) .

[3]. Dr.Roywinkelman , Director Produced by the Florida center for instructional Technology collage of education University of south Florida "what is a network?", http://www.fcit.usf.edu/ network/chap1.html.(2013).

[4]. Robert Hartman, AshishMalpani, ManjeshSiddamurthy, Tracy Alonzo, Colin King and Kevin Locklear, "Campus Networking Solutions Reference Architecture 1.0 (Large Campus)" .( March 2013).

\section{BIOGRAPHY}

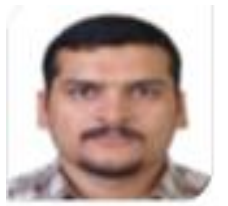

I wish to introduce myself as Ali Hussein Mousa, a prospective student aiming to get high academic education degree of Computer Engineering. I went on to pursue my Bachelor of Engineering with specialization in Software Engineering from a reputed University. I was quickly absorbing and assimilating information and knowledge in topics like Artificial Intelligence, Networks, and Internet Security. Permanently there is a connected line between my past hubby and present passions, so I feel proud and lucky that I presence in this scientific field to get advance and deeply knowledge. Now I work as a demonstrator in the Computer laboratory at Engineering college / University of Karbala / Iraq, My goal is to be with adequate knowledge and experience, hopping that I can grew up my own tiny spotlight in the road of acknowledgement of Computer Engineering. 